\title{
Throughput of the Multi-hop Slotted Aloha with Multi-packet Reception
}

\author{
M. Coupechoux ${ }^{1,2}$, T. Lestable ${ }^{1,3}$, C. Bonnet ${ }^{2}$, and V. Kumar ${ }^{1}$ \\ 1 Alcatel Research \& Innovation, route de Nozay, 91460 Marcoussis, France, \\ marceau. coupechoux@alcatel.fr, \\ 2 Institut Eurecom, Mobile Communications Dpt., Sophia-Antipolis, France, \\ 3 Supélec, Radio Dpt., Gif-sur-Yvette, France
}

\begin{abstract}
In this paper, we analyse the throughput of a multihop network, where nodes use slotted ALOHA as medium access protocol (MAC) and are able to receive simultaneously several packets in a slot. We provide a closed-form formula for the throughput in the generic case as a function of the probability, $r_{n, k}$, for a receiver to decode $k$ packets given that $n$ have been sent in its neighborhood. We then consider several simple models for the computation of the $r_{n, k}$, when spread slotted ALOHA is used. In particular, we compare the performances of a matched filter (MF) receiver with those of a linear minimum meansquare error (MMSE) multi-user detector (MUD). Capacity results show the great advantage of multi-packet reception and highlight the near-far resistance of the MUD scheme.
\end{abstract}

\section{Introduction}

In recent years, a lot of effort has been spent in the design of protocols for ad hoc networks. Such packet networks are multi-hop and operate without any fixed infrastructure. This can be a low cost and easily deployable technology to provide high speed Internet access in a wireless environment, to organize networks of sensors, or to complement the coverage of future cellular networks.

In this paper, we pay special attention to the MAC sub-layer and, in particular, to the traditional slotted ALOHA scheme. A lot of protocols have been proposed in the literature to address the issue of medium access, and we can distinguish two main families: the contention based schemes and the conflict-free schemes. Slotted ALOHA is the most simple protocol of the first category. Other examples are MACA [1], MACAW [2, FAMA [3], or IEEE 802.11 DCF 4]. On the other hand, conflict-free protocols allow the reservation of the channel for a certain amount of time and transmissions are then conflict-free. In this case, the reservation phase or the transmission of broadcast packets often relies on a slotted ALOHA scheme. That is the reason why this simple protocol is of great interest.

The spatial capacity of the slotted ALOHA protocol has been studied in [5], where the effect of capture is detailed. This capacity has been obtained with the assumption that receivers devices are able to decode at most a single 
packet per slot. However, research performed since the early 1980's in the domain of multi-user detection in CDMA systems [14 shows that this condition can be overcome. Indeed, receivers using multi-user detection schemes can decode the packets from several simultaneous transmitters. In particular, the near-far resistance of the multi-user detectors [13] makes this technique very attractive for ad hoc networks, where power control schemes are much more difficult to implement than in traditional single-hop systems.

In this paper, we extend the result of [5] in the case of multi-packet reception (section 2) and we provide a closed-form formula for the throughput of the slotted ALOHA as a function of the probability, $r_{n, k}$, for a receiver to decode $k$ packets given that $n$ have been sent in its neighborhood. In section 3 we detail three different models of multi-packet reception: a simple model often used in the literature, a bank of MF, and a MMSE multi-user detector. At last, in section 4, we provide numerical results and highlight the near-far resistance of the MUD scheme.

\section{Spatial Throughput with Multi-packet Reception}

\subsection{Models}

Throughout this paper, we will consider a packet radio network of nodes, spatially distributed in the plane according to a Poisson process with parameter $\lambda$. That means that the probability to find $k$ nodes in any region, $A$, of area $S(A)$ is:

$$
P[k \text { in } A]=\frac{(\lambda S(A))^{k}}{k !} e^{-\lambda S(A)} .
$$

We will assume that the considered network is large and we will neglect the edge effects.

All nodes are assumed to operate with a half-duplex radio device. This means that a collision of the second order can occur if a node receives a packet, while it is itself transmitting during the same slot. In this case, the packet is lost. The transmit power is constant and equals $P_{0}$.

As explained in the introduction, we assume that nodes access the channel by using the slotted ALOHA protocol, i.e., time is divided in equal time-slots. At a given slot, a node sends a packet with a fixed probability $p$. Otherwise, it is able to receive one or several packets coming from the transmitters. Let $R_{0}$ be the reception radius of a receiver. $R_{0}$ is the maximum distance from which can come a packet destined to this receiver. If there are $n$ transmitters within $R_{0}$ from the receiver, the probability to decode $k$ packets is $r_{n, k}$.

We assume that packets destined towards a particular node in the network are routed with equal probability towards one of the neighboring nodes that lies in the direction of the destination. All these assumptions are taken from [5].

First of all, we are interested in the local throughput of the system, i.e., the expected number of packet received per slot. We will then evaluate the expected forward progress of a packet and conclude our study with the total throughput of the network. 


\subsection{Preliminary Results}

Before looking at the local throughput, we recall two preliminary results already given in 5 . We consider a particular node $a$ and we define the random variable $X$ as the number of correctly decoded packets destined to $a$ in a given slot. Let us define two important events: $(A)$ the event that $a$ does not transmit; $(T)$ the event that a particular sender $t$ sends a packet to $a$. We have the two basic results:

$$
\begin{aligned}
& P[A]=1-p, \\
& P[T]=\frac{1-e^{-\lambda \pi R_{0}^{2} / 2}}{\lambda \pi R_{0}^{2}},
\end{aligned}
$$

where $p$ is the probability of transmission, $\lambda$ is the density of the nodes, and $R_{0}$ is the transmission range. Note that if nodes are spatially distributed according to a Poisson process with density $\lambda$, senders, at a given time-slot, are spatially distributed according to a Poisson process with density $\lambda p$ (see e.g. [16]).

\subsection{Local Throughput}

We are now in position to evaluate the local throughput. Let us define two more events: $\left(T_{n}\right)$ the event that there are $n$ senders in the neighborhood of $a ;\left(D_{k}\right)$ the event that $a$ decodes exactly $k$ packets in the given time-slot. Now, the probability that $a$ receives $x$ packets given $(A),\left(T_{n}\right)$, and $\left(D_{k}\right)$ is:

$$
P\left[X=x \mid A, T_{n}, D_{k}\right]=\left(\begin{array}{c}
k \\
x
\end{array}\right) P[T]^{x}(1-P[T])^{k-x}, k \geq x,
$$

because among the $k$ packets decoded, $x$ are destined to $a$. This probability is zero if $k<x$. We now successively un-condition this relation:

$$
\begin{aligned}
P\left[X=x \mid A, T_{n}\right] & =\sum_{k=0}^{n} P\left[X=x \mid A, T_{n}, D_{k}\right] P\left[D_{k} \mid A, T_{n}\right] \\
& =\sum_{k=0}^{n} P\left[X=x \mid A, T_{n}, D_{k}\right] r_{n, k} \\
& =\sum_{k=x}^{n}\left(\begin{array}{l}
k \\
x
\end{array}\right) P[T]^{x}(1-P[T])^{k-x} r_{n, k} .
\end{aligned}
$$

The second line is justified by the fact that the events $\left(D_{k}\right)$ and $(A)$ are independent. The third line takes into account Eq. 4 Now, assuming that the considered node $a$ does not transmit:

$$
P[X=x \mid A]=\sum_{n=0}^{\infty} P\left[X=x \mid A, T_{n}\right] P\left[T_{n} \mid A\right]
$$




$$
\begin{aligned}
& =\sum_{n=0}^{\infty} P\left[X=x \mid A, T_{n}\right] \frac{\left(\lambda p \pi R_{0}^{2}\right)^{n}}{n !} e^{-\lambda p \pi R_{0}^{2}} \\
& =\sum_{n=0}^{\infty} \sum_{k=x}^{n}\left(\begin{array}{l}
k \\
x
\end{array}\right) P[T]^{x}(1-P[T])^{k-x} r_{n, k} \frac{\left(\lambda p \pi R_{0}^{2}\right)^{n}}{n !} e^{-\lambda p \pi R_{0}^{2}}
\end{aligned}
$$

The second equation results from the fact that $\left(T_{n}\right)$ and $(A)$ are independent and that the density of the senders is $\lambda p$ as explained before. Note that if $a$ is a sender at the considered slot, $a$ cannot receive any packet because of the halfduplex nature of its radio device. So, for $x \neq 0$ :

$$
\begin{aligned}
P[X=x] & =P[X=x \mid A] P[A] \\
& =P[X=x \mid A](1-p),
\end{aligned}
$$

according to Eq2, We have obtained the probability distribution (pdf) function of $X$, the number of packets received by $a$ :

$$
P[X=x]=\sum_{n=0}^{\infty} \sum_{k=x}^{n}\left(\begin{array}{l}
k \\
x
\end{array}\right) P[T]^{x}(1-P[T])^{k-x} r_{n, k} \frac{\left(\lambda p \pi R_{0}^{2}\right)^{n}}{n !} e^{-\lambda p \pi R_{0}^{2}}(1-p) .
$$

The throughput in $a$ is immediatly obtained by taking the expectation of $X$ :

$$
E[X]=\sum_{x=1}^{\infty} x P[X=x]
$$

If there are $N$ nodes in the network, the local throughput, $S$, of the network, i.e., the throughput at the MAC layer is:

$$
S=N E[X]
$$

Note that the single-packet detection without capture is a special case of the aboves formulas. Indeed, by taking $r_{1,1}=1, r_{n, 0}=1$ for $n \neq 1$, and $r_{n, k}=0$ otherwise, we get:

$$
\begin{aligned}
E[X] & =P[X=1] \\
& =P[T]\left(\lambda p \pi R_{0}^{2}\right) e^{-\lambda p \pi R_{0}^{2}}(1-p) \\
& =p(1-p)\left(1-e^{-\lambda \pi R_{0}^{2} / 2}\right) e^{-\lambda p \pi R_{0}^{2}},
\end{aligned}
$$

which is in accordance with the results of [5]

\subsection{Expected Forward Progress}

The forward progress, $z$, of a successful packet is the distance covered over a single-hop in the direction of the final destination. It has been proven in 5$]$ that:

$$
E[z]=\int_{0}^{R_{0}} \frac{2 r d(r)}{\pi} d r
$$


where $d(r)$ is the pdf of the distance $d$ between a sender and a receiver for a successful transmission (event that we denote $(R)$ ). Let us evaluate $d(r)$ in the case of multi-packet reception:

$$
P\left[r \leq d \leq r+d r \mid R, T_{n}, D_{k}\right]=\frac{P\left[R \mid r \leq d \leq r+d r, T_{n}, D_{k}\right] P\left[r \leq d \leq r+d r \mid T_{n}, D_{k}\right]}{P\left[R \mid T_{n}, D_{k}\right]} .
$$

We now make the realistic assumption that if $k$ packets are decoded among $n$, the successful senders are the $k$ closest senders to the receiver. Under this assumption, for $n>1$ and $1 \leq k \leq n$ :

$$
\begin{gathered}
P\left[R \mid T_{n}, D_{k}\right]=\frac{k}{n} \\
P\left[R \mid r \leq d \leq r+d r, T_{n}, D_{k}\right]=\sum_{i=0}^{k-1}\left(\begin{array}{c}
n-1 \\
i
\end{array}\right)\left(\frac{r^{2}}{R_{0}^{2}}\right)^{i}\left(1-\frac{r^{2}}{R_{0}^{2}}\right)^{n-1-i}, \\
P\left[r \leq d \leq r+d r \mid T_{n}, D_{k}\right]=P[r \leq d \leq r+d r]=\frac{2 r}{R_{0}^{2}} d r .
\end{gathered}
$$

Eq.[18 is the proportion of successful transmissions during the considered slot. Eq.19] is justified by the fact that a transmission is successful at distance $r$ from the receiver iff there are at most $k-1$ senders in the disk of radius $r$. Moreover, the probability for a sender to be in this disk is $r^{2} / R_{0}^{2}$. It is straightforward to verify that the integration of Eq 19 over the disk of radius $R_{0}$ results in Eq 18 The last equation is the pdf of the distance between any node in the disk of radius $R_{0}^{2}$ and the receiver. It is possible to un-condition Eq17 by taking into account the Poisson distribution and the $r_{n, k}$ probabilities:

$$
\begin{gathered}
P\left[r \leq d \leq r+d r \mid R, T_{n}\right]=\sum_{k=0}^{n} P\left[r \leq d \leq r+d r \mid R, T_{n}, D_{k}\right] r_{n, k}, \\
P[r \leq d \leq r+d r \mid R]=\sum_{n=1}^{\infty} \sum_{k=0}^{n} P\left[r \leq d \leq r+d r \mid R, T_{n}, D_{k}\right] r_{n, k} P\left[T_{n}\right] \\
=\sum_{n=1}^{\infty} \sum_{k=0}^{n} P\left[r \leq d \leq r+d r \mid R, T_{n}, D_{k}\right] r_{n, k} \frac{\left(\lambda p \pi R_{0}^{2}\right)^{n}}{n !} e^{-\lambda p \pi R_{0}^{2}} .
\end{gathered}
$$

We can now conclude for $d(r)$ :

$$
d(r)=\sum_{n=1}^{\infty} \sum_{k=1}^{n} \sum_{i=0}^{k-1}\left(\begin{array}{c}
n-1 \\
i
\end{array}\right)\left(\frac{r^{2}}{R_{0}^{2}}\right)^{i}\left(1-\frac{r^{2}}{R_{0}^{2}}\right)^{n-1-i} \frac{2 n r}{k R_{0}^{2}} \frac{\left(\lambda p \pi R_{0}^{2}\right)^{n}}{n !} e^{-\lambda p \pi R_{0}^{2}} r_{n, k} .
$$

The numerical evaluation of Eq[16] implies the following integration:

$$
\int_{0}^{R_{0}} r^{2(i+1)}\left(1-\frac{r^{2}}{R_{0}^{2}}\right)^{n-1-i} d r=\sum_{j=0}^{n-1-i}\left(\begin{array}{c}
n-1-i \\
j
\end{array}\right) \frac{(-1)^{j}}{R_{0}^{2 j}} \int_{0}^{R_{0}} r^{2(i+j+1)} d r
$$




$$
=\sum_{j=0}^{n-1-i}\left(\begin{array}{c}
n-1-i \\
j
\end{array}\right) \frac{(-1)^{j} R_{0}^{2 i+3}}{1+2(i+j+1)} .
$$

As a consequence:

$$
E[z]=\sum_{n=1}^{\infty} \sum_{k=1}^{n} \sum_{i=0}^{k-1} \sum_{j=0}^{n-1-i}\left(\begin{array}{c}
n-1 \\
i
\end{array}\right)\left(\begin{array}{c}
n-1-i \\
j
\end{array}\right) \frac{4(-1)^{j} R_{0} r_{n, k} e^{-\lambda p \pi R_{0}^{2}}\left(\lambda p \pi R_{0}^{2}\right)^{n}}{k \pi(3+2 i+2 j)(n-1) !}
$$

With the help of a software of formal computations, we can simplify this expression in:

$$
E[z]=\frac{4 R_{0} e^{-\lambda p \pi R_{0}^{2}}}{\pi} \sum_{n=1}^{\infty} \sum_{k=1}^{n} \frac{\Gamma\left(k+\frac{3}{2}\right)\left(\lambda p \pi R_{0}^{2}\right)^{n} r_{n, k}}{3 \Gamma\left(n+\frac{3}{2}\right) k !},
$$

where $\Gamma$ is the gamma function.

\subsection{End-to-End Throughput}

According to [5], for any randomly selected terminal, the expected path length between it and another selected terminal is given as $D=(128 / 45 \pi) \sqrt{N / \lambda \pi}$, where $N$ is the number of nodes in the network. Thus, the mean number of hops for a packet is $D / E[z]$ and the end-to-end throughput of the network per slot is:

$$
t=\frac{S E[z]}{D} .
$$

\section{Multi-packet Reception Models}

In this section, we assume that the previously considered ALOHA protocol is a spread slotted ALOHA. At a given time-slot, all senders are supposed to choose at random a pseudo-noise $(\mathrm{PN})$ code among a large book of low cross-correlated PN codes with spreading factor $L$, large. All potential receivers, i.e., all nodes have the knowledge of this book and are able to perform mulit-packet reception. We neglect the probability that two neighboring senders choose the same code in order to simplify the calculations. From the presented models, we derive values for the $r_{n, k}$.

\subsection{Simple Model}

The first model is a very simple one, often used in the literature, e.g., in [9]. It states that all of the simultaneous transmissions can be successfully received if no more than $K$ users are transmitting at the same time. If there are more than $K$ users transmitting at the same time, the multi-user receiver is overhelmed and a collision occurs. Thus:

$$
r_{n, k}=\left\{\begin{array}{l}
1, \text { if } k=n \text { and } n \leq K \\
1, \text { if } k=0 \text { and } n>K \\
0, \quad \text { otherwise }
\end{array}\right.
$$


In the following two models, a packet is assumed to be decoded by an idle node if its signal to interference plus noise ratio (SINR) reaches a SINR target at the output of the detector.

\subsection{Receiver with a Bank of Matched Filters}

In this section, we suppose that radio receivers devices are made of a bank of MF that are able to decode each spreading code individually. If $P_{0}$ is the transmit power, the received power at a distance $r$ is assumed to be $P(r)=P_{0} / r^{\gamma}$, where $\gamma>2$ is the path loss exponent. This expression is a far-field approximation that doesn't hold for small values of $r$. A packet is considered to be decoded if the SINR, $\beta$, of a signal at the output of the MF reaches a SINR target $\beta_{0}$, i.e., if:

$$
\beta=\frac{P(r)}{\sigma^{2}+\frac{1}{L} \sum_{i=0}^{n-1} \frac{P_{0}}{r_{i}^{\gamma}}} \geq \beta_{0},
$$

where $\sigma^{2}$ is the power of the noise, $n$ is the number of interferers, and $L$ is the spreading length.

In order to analitycaly evaluate the $r_{n, k}$ parameters, the cumulative distribution function (cdf) of the SINR is needed in the case of a Poisson field of interferers. This problem has been treated in [6] and in [11, where the characteristic function of the interference $Y=\sum_{i=0}^{n-1} P_{0} / r_{i}^{\gamma}$ has been obtained:

$$
\phi_{Y}(\omega)=\exp \left(-\pi \lambda p \Gamma(1-2 / \gamma) e^{-i \pi / \gamma} \omega^{2 / \gamma}\right), \omega \geq 0 \text { and } \gamma>2 \text {, }
$$

where $\Gamma$ is the gamma function and $p$ is the probability of transmission. This expression leads to the exact cdf of $\beta$ and thus to the $r_{n, k}$ in the MF case. However, we will see that this is not the case for the MUD receiver. That is the reason why we evaluate the $r_{n, k}$ probabilities thanks to Monte Carlo simulations in order to allow a fair comparison with the MMSE detector.

A Poisson field of interferers with density $\lambda p$ is generated on a two dimensional squared network $[-X \max ; X \max ] \times[-Y \max ; Y \max ]$. The considered receiver, $a$, is placed at $(0 ; 0) . R_{0}$ is fixed as the maximum distance from which can come packets for the receiver. In the abscence of interferer, $R_{0}$ verifies the following expression: $\beta_{0}=P_{0} /\left(R_{0}^{\gamma} \sigma^{2}\right)$. $n$ is the number of senders inside the disk of radius $R_{0}$ with center $a$. For each of these senders, the SINR is computed after summing the interference from the whole network. If the SINR reaches the SINR target, the packet from this sender is assumed to be decoded. A snapshot of the simulation is shown on Fig.1. Tab 1 shows the parameter values used for our simulations.

Fig.2 shows the plot of the matrix $r_{n, k}$ for $n \leq 14$ and $p=0.2$. The mean number of senders in the disk of radius $R_{0}$ is $\lambda p \pi R_{0}^{2} \simeq 5$, so the probability that $n>14$ is very low. This figure shows that for small values of $n$, all packets are decoded. Then, when $n$ increases, the number of decoded packets decreases. 


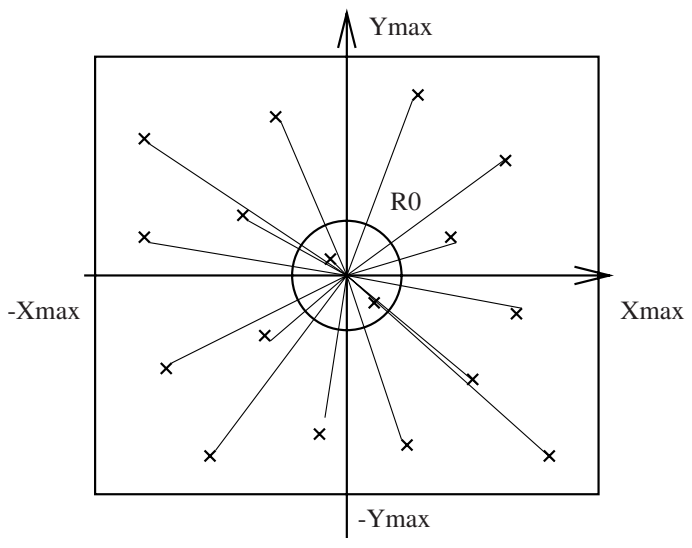

Fig. 1. Snapshot of the Monte Carlo simulation: the power of all the interferers are sumed at the receiver.

Table 1. Parameter values used for the Monte Carlo simulation in the case of MF receivers

\begin{tabular}{|c|c|}
\hline Parameter & Value \\
\hline \hline$X \max$ & 50 \\
\hline$Y \max$ & 50 \\
\hline$\lambda$ & 0.25 \\
\hline$p$ & 0.2 \\
\hline$L$ & 32 \\
\hline$P_{0}$ & 5 \\
\hline$\beta_{0}$ & 0.025 \\
\hline$\sigma^{2}$ & 0.2 \\
\hline$\gamma$ & 4 \\
\hline
\end{tabular}

\subsection{Receiver with MMSE Multi-user Detector}

In this section, we assume that receivers are able to perform multi-user detection thanks to a MMSE detector. While the traditional MF or Rake receiver treats interference from other users as noise, the MUD scheme jointly decodes all users.

The condition of decoding of a packet is still based on the SINR at the output of the signal detector. According to [15], to check if the target for a given sender's SINR, $\beta_{0}$, can be met for a given system of senders, it suffices to check the following condition:

$$
\frac{P}{\sigma^{2}+\frac{1}{L} \sum_{i=0}^{n-1} I\left(P_{i}, P, \beta_{0}\right)} \geq \beta_{0},
$$

where $P=P_{0} / r^{\gamma}$ is the received power of the given sender, $P_{i}$ is the received power from the interferer $i$ and $I\left(P_{i}, P, \beta_{0}\right)$ is the effective interference of sender 


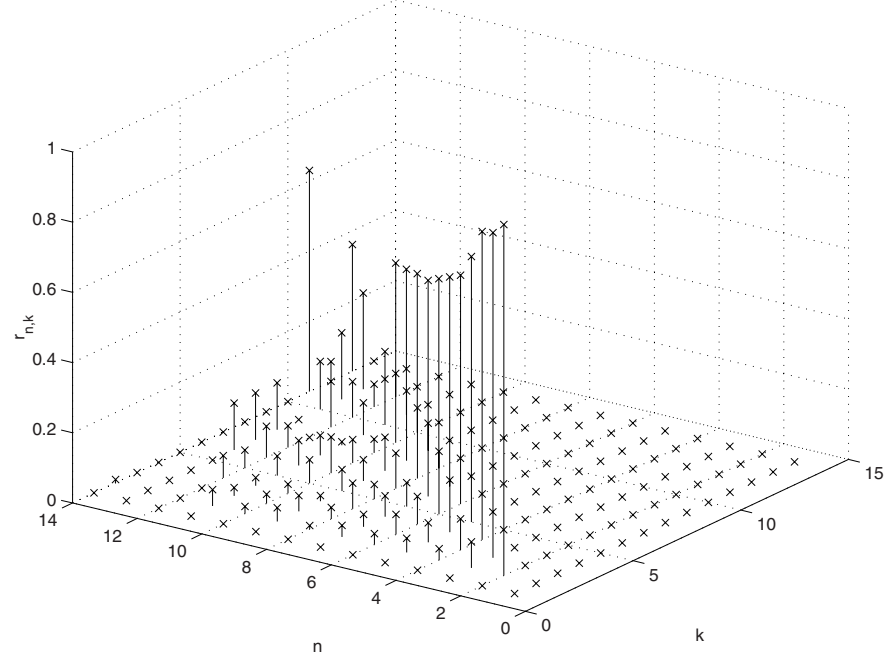

Fig. 2. Probabilities, $r_{n, k}$, for a receiver to decode $k$ packets given that $n$ have been sent in the case of a bank of MF.

$i$ on the considered sender at the target $\operatorname{SINR} \beta_{0}$ :

$$
I\left(P_{i}, P, \beta_{0}\right)=\frac{P P_{i}}{P+P_{i} \beta_{0}} .
$$

Eq.31 also used in 8 in the context of call admission control, is an approximation since it is true for large systems, when $L \rightarrow \infty, n \rightarrow \infty$ and $L / n=\alpha$, and for random spreading sequences.

We can show that the characteristic function of the interference for a given sender and a given SINR target, $\beta_{0}$ is:

$$
\phi_{Y}(\omega)=\exp \left(i \lambda p \pi \omega \int_{0}^{P / \beta_{0}}\left(\frac{P_{0}}{t}-\frac{P_{0} \beta_{0}}{P}\right)^{2 / \gamma} e^{i \omega t} d t\right) .
$$

While Eq[30 is seen as the characteristic function of a stable law, Eq 33 seems to be un-tractable for further computations. That is the reason why we rely on Monte Carlo simulations as explained in the previous section. Parameter values are given in Tab.1 and the condition of packet decoding is given by Eq 31 Fig.3 shows the graph of the matrix $r_{n, k}$ for $n \leq 14$. It is clear that the MUD scheme offers much better performances than the MF decoding. Note also that the simple model is a approximation of the MMSE performance if $K$ is chosen appropriately. 


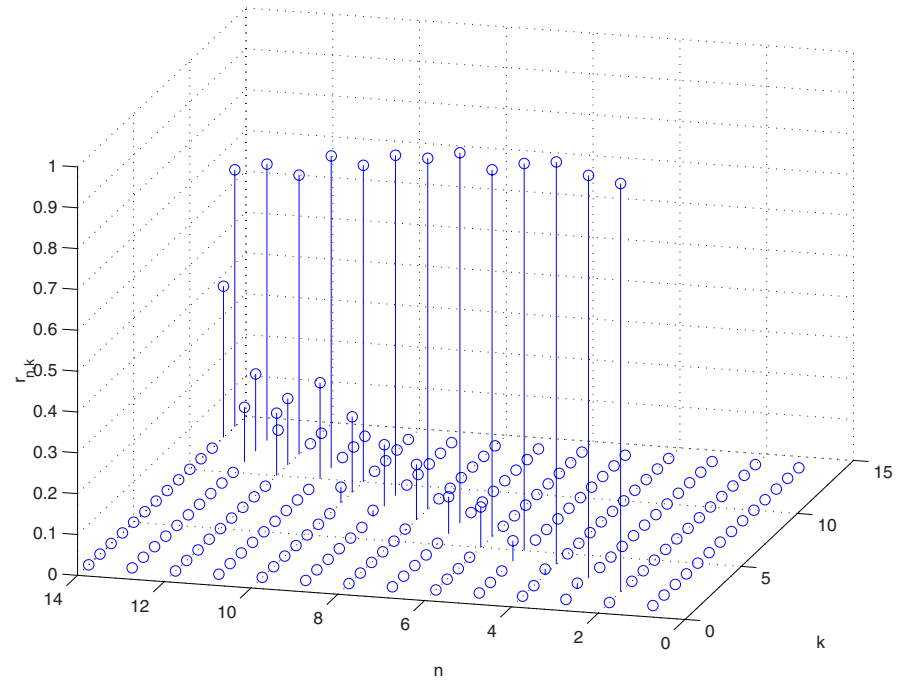

Fig. 3. Probabilities, $r_{n, k}$, for a receiver to decode $k$ packets given that $n$ have been sent in the case of MMSE MUD.

\section{Numerical Results}

In this section, we give numerical results for the three models of multi-packet reception presented previously. We focus our attention on the local throughput and on the end-to-end throughput of the network.

On Fig.4, we present the local throughput for the first simple model with different values of $K$. We observe in all cases the characteristic shape of the throughput of the ALOHA protocol as a function of the input load. As expected, the multi-packet reception feature improves the maximum achievable throughput.

Fig. 5 shows the end-to-end throughput for the first simple model with different values of $K$. Here also, we see the advantage of multi-packet reception. Note that the optimum probability of transmission depends on $K$. For $K=1$, we observe the classical result that $p$ is optimum for $p=1 /\left(\lambda \pi R_{0}^{2}\right)$, which here is approximatly 0.05 . As $K$ increases, $p$ also increases because more packets can be handled by the receiver.

Fig.6 compares the local throughput of the MF receiver with this of the MMSE receiver. We observe the great advantage of the MUD over the conventional receiver (approximatly 30\% in our scenario). This advantage can also be seen on Fig.7, that shows the end-to-end throughput. Indeed, the joint detection of all users makes the MUD very robust to near-far problems. This near-far resistance is of great interest in ad hoc networks because power control schemes are difficult to implement in such decentralized networks. 


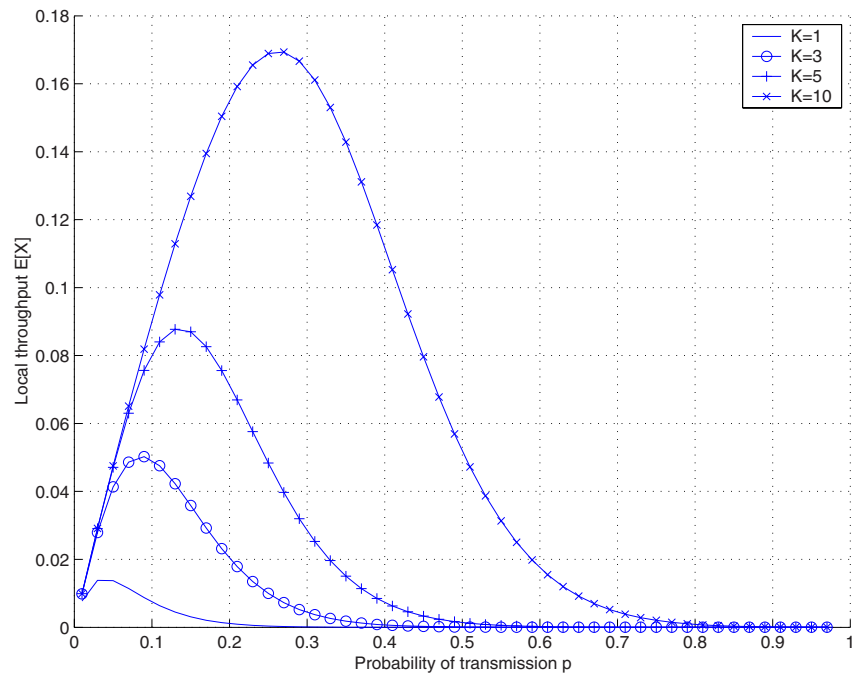

Fig. 4. Local throughput in packets/time-slot for the simple model of multi-packet reception for different values of $K$, the maximum number of packets that can be decoded by the receiver.

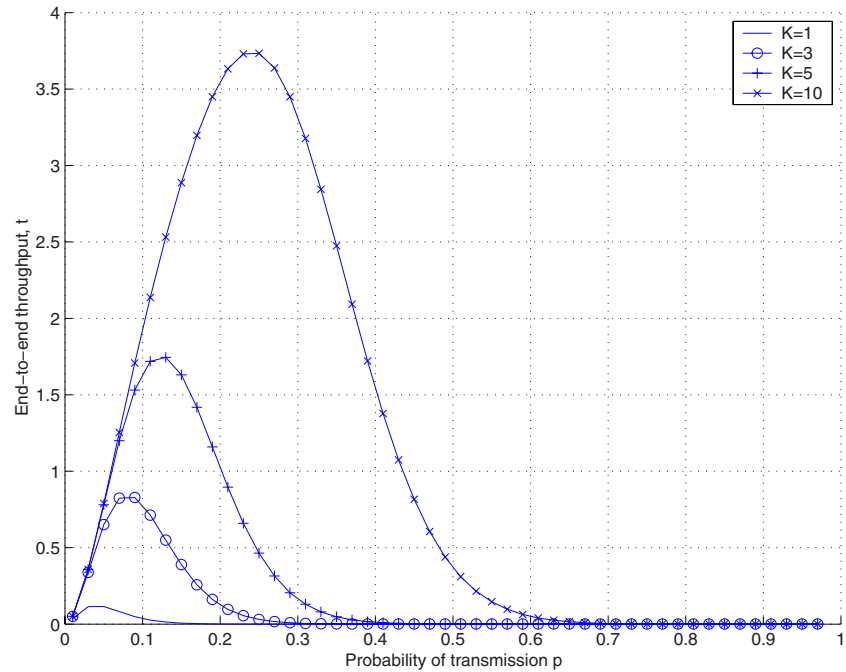

Fig. 5. End-to-end throughput in packets/time-slot for the simple model of multipacket reception for different values of $K$, the maximum number of packets that can be decoded by the receiver, and $N=100$ nodes. 


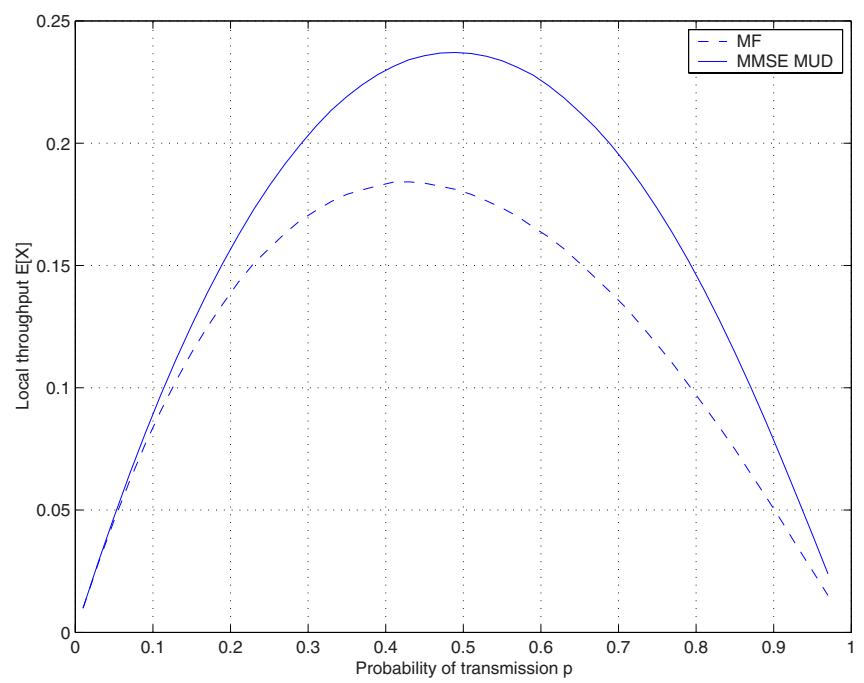

Fig. 6. Local throughput in packets/time-slot for the MF receiver and the MMSE receiver.

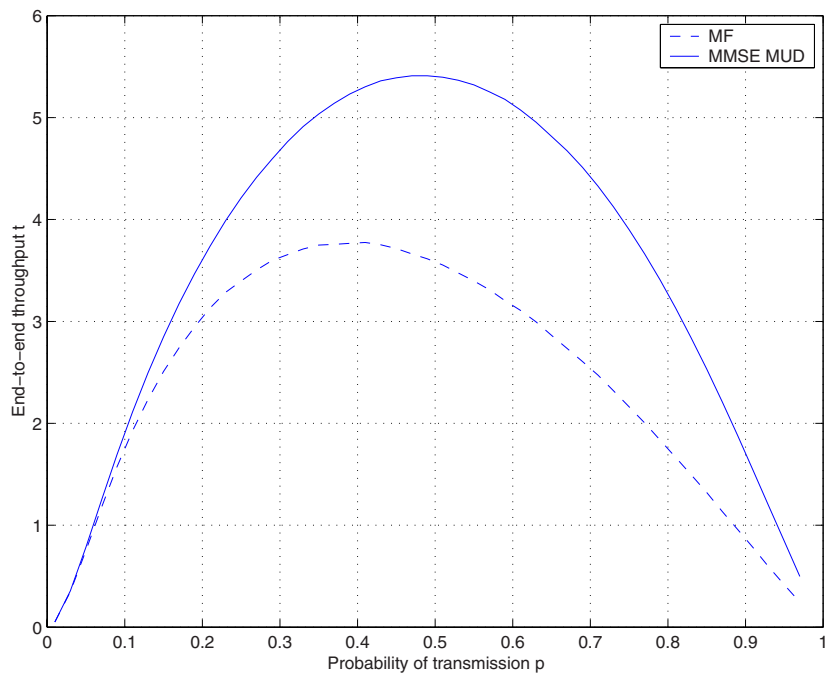

Fig. 7. End-to-end throughput in packets/time-slot for the MF receiver and the MMSE receiver for $N=100$ nodes. 


\section{Conclusion}

In this paper, we have analyzed the throughput of the slotted ALOHA protocol in a multi-hop network, where nodes are able to perform multi-packet reception. We have derived from an analytical study a closed-form formula for the local throughput and the end-to-end throughput of such a network. This formula is given as a function of the probabilities, $r_{n, k}$, of decoding $k$ packet when $n$ senders have transmitted a packet in the neighborhood of the receiver. Then, three models of multi-packet reception have been presented in the case of CDMA systems: a simple one, often used in the literature, and two models based on two types of receivers, i.e., a bank of matched filters and a MMSE MUD detector. In the latter case, we have provided the characteristic function of the interference. However because of the un-tractability of this formula, we relied on Monte Carlo simulations in order to evaluate the $r_{n, k}$ probabilities. Numerical results show the great advantage of the near-far resistance of the MMSE receiver.

\section{References}

1. P. Karn, MACA - a New Channel Access Method for Packet Radio, Proc. of ARRL/CRRL, April 1990.

2. V. Bhargavan, A. Demers, S. Shenker, and 1. Zhang, MACAW: A Media Access Protocol for Wireless LAN's, Proc. of ACM SIGCOMM, pp 212-225, Aug. 1994.

3. J. J. Garcia-Luna-Aceves ans C. L. Fullmer Floor Acquisition Multiple Access (FAMA) in Single-Channel Wireless Networks Mobile Networks Applications, vol. 4, pp 157-174, Baltzer Science Publishers, 1999

4. IEEE P802.11, Draft Standard for Wireless LAN: Medium Access Control (MAC) and Physical Layer (PHY) Specifications IEEE, July 1996.

5. R. Nelson and L. Kleinrock The Spatial Capacity of a Slotted ALOHA Multihop Packet Radio Network with Capture, IEEE Trans. On Communications, VOL. COM-32, NO. 6, June 1984

6. E. S. Sousa and J. A. Silvester, Optimum Transmisssion Ranges in a DirectSequence Spread-Spectrum Multihop Packet Radio Network, IEEE Journal On Selected Areas in Communications, VOL. 8, N0. 5, June 1990

7. E. S. Sousa, Performance of a Spread Spectrum Packet Radio Network Link in a Poisson Field of Interferers, IEEE Trans. On Information Theory, VOL. 38, NO. 6, Nov. 1992

8. C. Sankaran and A. Ephremides, The Use of Multiuser Detectors for Multicasting in Wireless Ad Hoc CDMA Networks, IEEE Trans. On Information Theory, VOL. 48, NO. 11, Nov. 2002

9. Q. Liu, E.-H. Yang, and Z. Zhang, Throughput Analysis of CDMA Systems Using Multiuser Receivers, IEEE Trans. On Communications, VOL. 49, NO. 7, July 2001

10. A. Polydros and J. Silvester, Slotted Random Access Spread-Spectrum Networks: An Analytical Framework, IEEE Journal On Selected Areas in Communications, VOL. 5, NO. 6, July 1987

11. E. S. Sousa, Interference Modeling in a Direct-Sequence Spread-Spectrum Packet Radio Network, IEEE Trans. On Communications, VOL. 38, NO. 9, Sept. 1990

12. J. Q. Bao and L. Tong, A Performance Comparison Between Ad Hoc and Centrally Controlled CDMA Wireless LANs, IEEE Trans. On Wireless Communications, VOL. 1, NO. 4, Oct. 2002 
13. R. Lupas and S. Verd, Near-Far Resistance of Multiuser Detectors in Asynchronous Channels, IEEE Trans. On Communications, VOL. 38, NO. 4, Apr. 1990

14. S. Verdu, Multiuser Detection Cambridge University Press, 1998

15. D. N. C. Tse and S. V. Hanly, Linear Multiuser Receivers: Effective Interference, Effective Bandwidth and User Capacity, IEEE Trans. On Information Theory, VOL. 45, NO. 2, Mar. 1999

16. A. Frey and V. Schmidt, Marked Point Processes in the Plane I, Advances in Performance Analysis, VOL. 1, Notable Publications Inc., 1998 